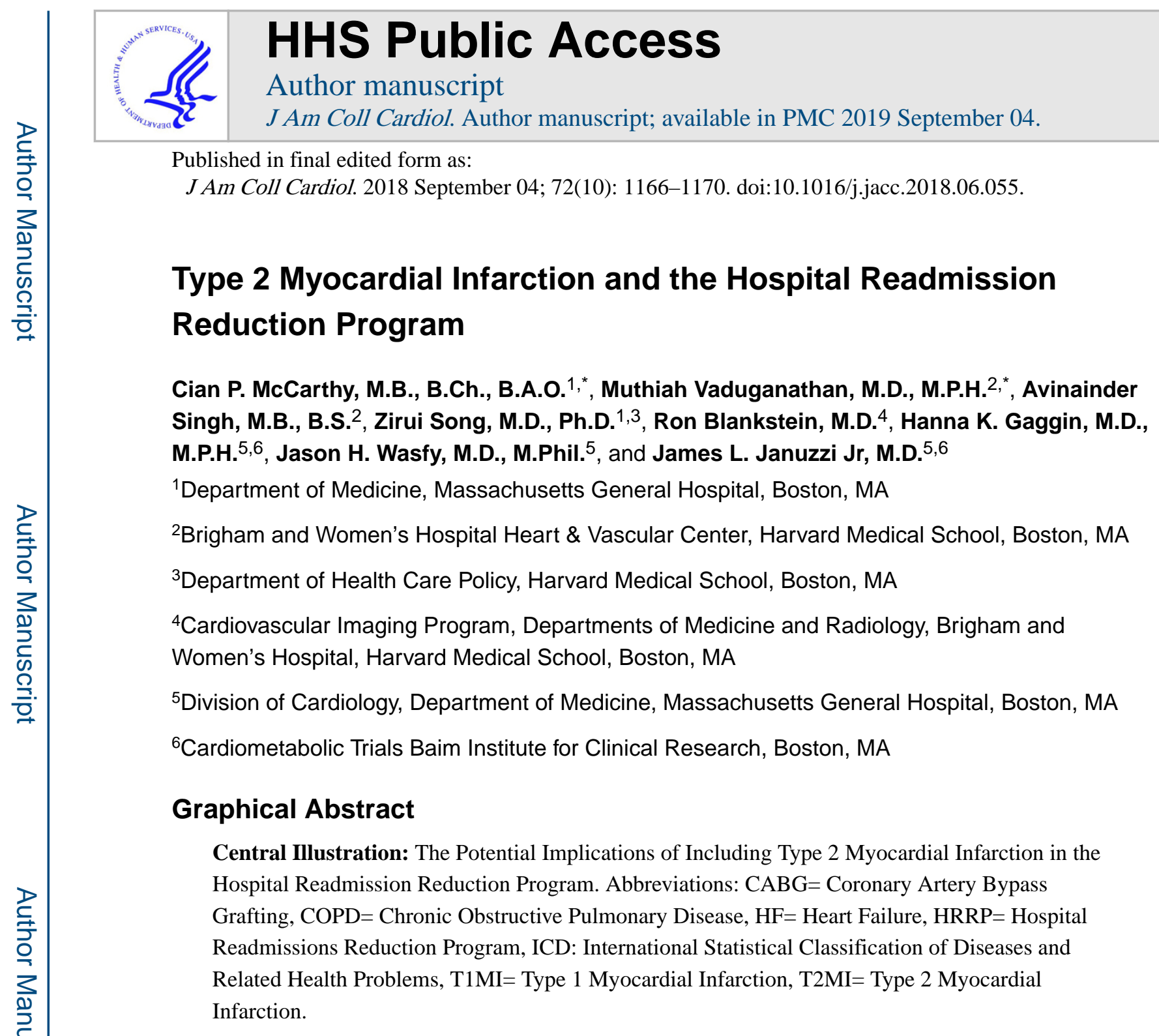

Correspondence: James L. Januzzi Jr, MD, Massachusetts General Hospital, 32 Fruit Street, Yawkey 5B, Boston, MA, 02114, P: +1-617-726-3443, F: +1-617-643-1620, jjanuzzi@ mgh.harvard.edu.

*Both authors contributed equally

Tweet:

Is it time to exclude Type 2 MI patients from the Hospital Readmission Reduction Program? @JJheart_doc @CianPMcCarthy

@ mvaduganathan 


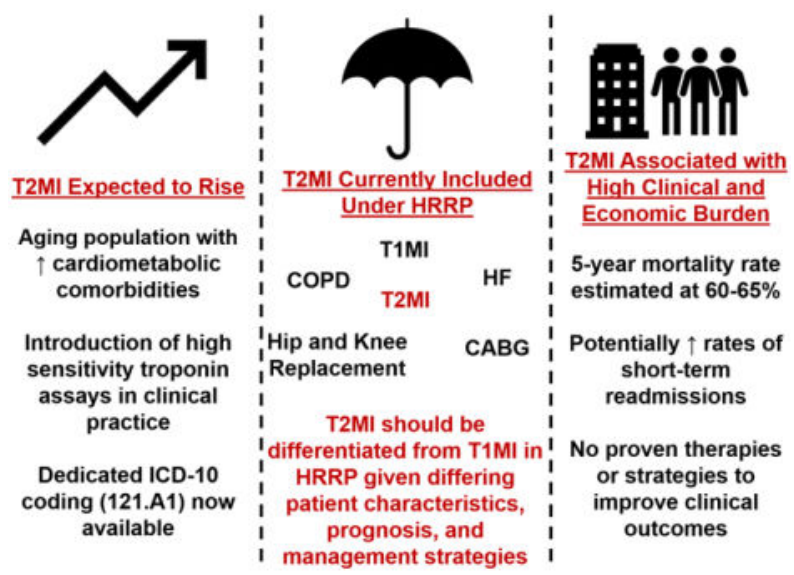

In 2012, the Centers for Medicare \& Medicaid Services (CMS) launched the Hospital Readmissions Reduction Program (HRRP) with the goal of reducing 30-day readmissions in the Medicare population (1). The objective of the program is to reduce Medicare spending by preventing avoidable repeat hospitalizations, and to improve the quality of post-acute care. Acute myocardial infarction (MI), a principal target of the program, is one of six medical conditions included, contributing to a total of $\$ 528$ million withheld from hospitals under the HRRP in fiscal year 2017.

Acute MI is a broad diagnosis, encompassing a diverse cohort of patients with varying precipitating factors and causal mechanisms of cardiac injury. Recognizing this, in 2007, a Task Force for the Redefinition of MI developed the Universal Definition of MI consensus document, which introduced 5 subtypes of MI (2); the most frequently encountered are type $1 \mathrm{MI}$ (characterized by atherosclerotic processes such as plaque rupture, ulceration, fissuring, erosion, or dissection resulting in coronary thrombosis) and type $2 \mathrm{MI}$ (myocardial necrosis resulting from a mismatch in myocardial oxygen supply-demand and occurring in the absence of atherothrombosis including coronary artery embolism, vasospasm, and spontaneous coronary artery dissection) (2).

Despite differences between type 1 and type 2 MI (Table 1), both are included under the HRRP, and it is reasonable to suspect that readmission following type 2 MI contributes a substantial amount of penalty funds under the law. Few data are available to date, but at least $10 \%$ of all readmitted patients (cardiovascular and non-cardiovascular patients) that have actually contributed to the HRRP penalty may have type 2 MI (3). Until recently, it was not possible to differentiate patients with type 1 and type 2 MI using administrative data due to the absence of an International Statistical Classification of Diseases and Related Health Problems (ICD) code for each subtype of MI. Data suggest approximately $90 \%$ of type 2 MI cases were not being coded (4). If coded correctly, cases of type 2 MI may actually outnumber cases of type $1 \mathrm{MI}$ (4). However, in the absence of strict diagnostic criteria for type $2 \mathrm{MI}$, a diagnosis of type $2 \mathrm{MI}$ is subject to ambiguity.

Given differences in the ramifications of type 1 MI compared with type 2 MI, in October 2017, an ICD code for type 2 MI was introduced for the first time. Patients who experience type $2 \mathrm{MI}$ represent a heterogeneous cohort, with relatively higher mortality rates when 
compared with those with type $1 \mathrm{MI}(5,6)$. With the regulatory approval and imminent introduction of high sensitivity troponin assays across the U.S., the prevalence of detectable myocardial injury-including that from type $2 \mathrm{MI}$-is expected to increase (7). In the context of these nationwide changes, the potential uncertainties and implications of including type 2 MI patients in the HRRP warrant consideration by clinicians and policymakers (Central Illustration).

\section{The Hospital Readmissions Reduction Program}

Under the HRRP (1), hospitals must publicly report 30-day admission rates, which are then compared to risk-standardized readmission rates; financial penalties are incurred if these rates are exceeded (1). Financial penalties levied against hospitals were introduced on October $1^{\text {st }}, 2012$, with penalties initially up to $1 \%$ of total Medicare reimbursement, and rising up to $3 \%$ in subsequent years (1).

\section{Type 2 Myocardial Infarction: Patient Characteristics and Prognosis}

Patients who suffer type 2 MI encompass a diverse cohort and are typically older with greater medical complexity compared with patients with type 1 MI $(6,8)$. For example, these patients have a higher prevalence of hypertension, hyperlipidemia, anemia, chronic kidney disease, and prior stroke compared with patients with type 1 MI (5). Type 2 MI more commonly occurs in the context of hemodynamic instability, tachycardia, hypoxemic respiratory failure, decompensated heart failure, and recent surgery. Significant coronary artery disease is less prevalent in patients with type $2 \mathrm{MI}$ when compared with type $1 \mathrm{MI}$ $(55 \%$ versus $88 \%, \mathrm{P}<0.001)(8)$.

The post-discharge prognosis of patients with type $2 \mathrm{MI}$ is expectedly poor. In a large retrospective study by Chapman and colleagues of 2,122 patients with an elevated troponin level, all-cause mortality was higher in patients with type 2 MI (63\%) than in patients with type $1 \mathrm{MI}(37 \%)$ at 5 years (5). Excess deaths in those with type 2 MI were more often due to non-cardiovascular causes compared with those with type $1 \mathrm{MI}$. The observed crude major adverse cardiovascular events (MACE) rates were similar between groups (5). Similarly, Sarkisian et al. reported type 2 MI patients had a mortality rate of $63 \%$ over a 3.2year follow-up period (9). In a prospective study, Gaggin and colleagues reported patients with type $2 \mathrm{MI}$ had higher rates of subsequent adverse events than those without type $2 \mathrm{MI}$ (per 100 person-years: MACE, 54 versus 21, $\mathrm{P}<0.001$; all-cause death, 23 versus $3, \mathrm{P}<0.001$; cardiovascular death, 18 versus $3, \mathrm{P}<0.001$; heart failure events, 22 versus $7, \mathrm{P}<0.001$ ) (6).

Few studies have examined readmission rates after type $2 \mathrm{MI}$, but those that have suggest such readmissions are not insignificant. In 998 cases of type 2 MI in the Calgary Health Region, readmission rates were high: $14 \%$ at 30 days, and 35\% at 1 year (10). Smilowitz et al. examined the frequency of readmission after perioperative MI in 8,085 patients included in the 2015 U.S. Nationwide Readmission Database; many cases were suspected to be type 2 MI as revascularization rates were low (16\%). The investigators found readmission rates were high at $19 \%$, with a median cost of hospital readmission of $\$ 9,893$. Approximately $40 \%$ of readmissions were due to infectious or bleeding complications, and non- 
cardiovascular causes of rehospitalization outnumbered those due to cardiovascular reasons (11). Similarly, examining 30-day readmissions after type 2 MI, Martin and colleagues found that greater than a quarter of readmissions were due to infection and another quarter due to non-cardiac chest pain (3).

A major challenge confronting clinicians managing patients with type $2 \mathrm{MI}$ is ambiguity in management options for affected patients. In stark contrast with type $1 \mathrm{MI}$, which has a clear set of guideline-based recommendations for treatment, routine management of type 2 MI remains uncertain. Whether therapies known to reduce risk in type $1 \mathrm{MI}$ (such as antiplatelet drugs, statins, beta-blockers, or coronary revascularization) have similar benefits for those with type 2 MI is poorly understood.

\section{A Path Forward}

Studies to understand optimal care of patients with type $2 \mathrm{MI}$ are greatly needed as these patients are less likely to receive cardioprotective therapies when compared with those experiencing type $1 \mathrm{MI}$ (10), albeit, with the caveat that these medications are, thus far, of unproven benefit in this cohort due to an absence of randomized clinical trials. It is plausible that including the diagnosis of type $2 \mathrm{MI}$ in the HRRP may encourage further research to identify optimal treatment strategies that result in improving post-hospitalization management. Nevertheless, we articulate concerns regarding the inclusion of type 2 MI under the HRRP:

1. The reported incidence of type $2 \mathrm{MI}$ is expected to increase for $\mathbf{2}$ reasons: Prior to the introduction of a specific ICD 10 code, administrative coding of type 2 MI was highly variable and often infrequent (4). Retrospective studies report that type 2 MI patients made up 14-21\% of ICD-coded MI cases $(4,12)$. However, approximately $90 \%$ of type 2 MI cases were not being coded (4). Second, with widespread introduction of high sensitivity troponin assays in the U.S., the detection of myocardial injury due to MI as well as non-MI mechanisms may increase. Without appropriate education, many patients with myocardial injury driven by non-coronary mechanisms may be incorrectly labelled as having a type $2 \mathrm{MI}$ (for which demonstrable ischemia is a prerequisite). Given the current penalization implications, it is crucial that physicians and those involved in diagnostic coding differentiate type 1 and 2 MI from myocardial injury.

2. Targeting readmissions related to type 2 MI may not necessarily improve global processes of care and clinical outcomes: Early analyses of the performance of HRRP has demonstrated a reduction in readmission rates among MI patients, but this did not correlate with improvements in quality of care or long-term outcomes (13). By incentivizing readmission reduction, necessary readmissions in more complex patients with type 2 MI may be inappropriately avoided.

3. Inclusion of type $2 \mathrm{MI}$ in HRRP has the potential to further worsen penalty inequalities influencing safety-net hospitals (13), and may discourage access 
and care of certain low income or minority populations: Older patients with high comorbid burden at risk for type 2 MI may more frequently present to these hospital systems, which disproportionately bear financial penalties in the current system.

4. Expected risk of readmission after type 2 MI may be underestimated: During the period when the risk standardized 30-day readmission rates for acute MI were adjudicated using claims data (July 2008 through June 2011), diagnosis of type 2 MI was in its infancy, having only been introduced in 2007. Further, there was no specific ICD code for this cohort at the time, and the majority of type 2 MI patients may not have been coded as an MI (4). As such, they were likely underrepresented, or at least variably included, in risk estimates.

5. Undercoding of type $2 \mathrm{MI}$ in administrative data is a concern: Physicians may be discouraged from classifying a patient with a diagnosis of type 2 MI due to the penalization implications of possible readmission. Without accurate coding, informative data collection, and improvements in processes of care for these patients will be challenging. Further, subsequent physicians caring for the patient may not recognize the severity of their illness if the diagnosis was not correctly recorded.

6. Lastly, and perhaps most importantly, the primary objective of the HRRP is to reduce preventable hospital readmissions: It bears repeating that unlike standardized, clear, guideline-directed approaches to the management of type 1 MI, there are currently no evidence-based treatments options for patients with type $2 \mathrm{MI}$ or any guideline or consensus statements on how these patients should be managed. Thus, it seems unreasonable to hold hospitals accountable for readmission of these patients, in the absence of strategies to prevent repeat hospitalization. Indeed, many patients with type $2 \mathrm{MI}$ will be readmitted for noncardiovascular reasons related to their comorbidities $(3,11)$.

\section{Conclusion}

With the recent introduction of a specific ICD code, the decision to include patients with type $2 \mathrm{MI}$ under the HRRP is at a crossroads. While encouraging clinical research and changing payment to reward more efficient spending for these patients is welcome, high financial penalties, inaccurate readmission risk estimates, and an absence of evidence-based treatment options raise concerns for potential unintended consequences. As a result, we believe patients with type 2 MI should be differentiated from those with type $1 \mathrm{MI}$ in the HRRP recognizing divergence in patient characteristics, prognosis, and management strategies.

\section{Disclosures:}

Dr. McCarthy has no conflict of interest to disclose.

Dr. Vaduganathan is supported by the NHLBI T32 postdoctoral training grant (T32HL007604).

Dr. Singh has no conflict of interest to disclose. 
Dr. Song is supported by the Office of the Director, National Institutes of Health (NIH Director's Early Independence Award No. 1DP5OD024564-01).

Dr. Blankstein has served on the advisory board of Amgen; and recieves research support from Amgen Inc and Astellas Inc.

Dr. Gaggin is supported in part by the Clark Fund for Cardiac Research Innovation; received grant support and consulting income from Roche Diagnostics; research payments for clinical endpoint committees from Radiometer.

Dr. Wasfy served on the Health Care Payment Learning and Action Network committee on cardiac episode payment, which provided recommendations to Centers for Medicare and Medicaid Services about episode payments for cardiac conditions. Dr. Wasfy also reports a career development award from Harvard Catalyst and the National Institutes of Health (KL2 TR001100).

Dr. Januzzi is supported in part by the Hutter Family Professorship; has received grant support from Abbott, Cleveland Heart Labs, Singulex, and Prevencio; has received consulting income from Roche Diagnostics, Critical Diagnostics, and Novartis; and has participated in clinical endpoint committees/data or safety monitoring boards for Novartis, Amgen, GE, Janssen, Pfizer, and Boehringer Ingelheim.

\section{Abbreviations:}

CMS

HRRP

ICD

MACE

MI
Centers for Medicare \& Medicaid Services

Hospital Readmissions Reduction Program

International Statistical Classification of Diseases and Related Health Problems

Major adverse cardiovascular events

Myocardial infarction

\section{References}

1. Centers for Medicare \& Medicaid Services. Readmissions Reduction Program (HRRP). Available at: http://go.cms.gov/1eTMN74. Accessed May 15th 2018.

2. Thygesen K, Alpert JS, White HD et al. Universal definition of myocardial infarction. Circulation. 2007;116:2634-53. [PubMed: 17951284]

3. Martin LM, Thompson RW, Ferris TG et al. Abstract 014: Evaluation of Readmission after Acute Myocardial Infarction for Patients Included in Medicare's Hospital Readmissions Reduction Program. Circ Cardiovasc Qual Outcomes. 2017;10:A014-A014.

4. Diaz-Garzon J, Sandoval Y, Smith SW et al. Discordance between ICD-Coded Myocardial Infarction and Diagnosis according to the Universal Definition of Myocardial Infarction. Clin Chem. 2017;63:415-419. [PubMed: 27811209]

5. Chapman AR, Shah ASV, Lee KK et al. Long Term Outcomes in Patients with Type 2 Myocardial Infarction and Myocardial Injury. Circulation 2018;137:1236-1245. [PubMed: 29150426]

6. Gaggin HK, Liu Y, Lyass A et al. Incident Type 2 Myocardial Infarction in a Cohort of Patients Undergoing Coronary or Peripheral Arterial Angiography. Circulation 2017;135:116-127. [PubMed: 27881568]

7. Januzzi JL, Sandoval Y. The Many Faces of Type 2 Myocardial Infarction. J Am Coll Cardiol. 2017;70:1569-1572. [PubMed: 28935033]

8. Saaby L, Poulsen TS, Hosbond S et al. Classification of myocardial infarction: frequency and features of type 2 myocardial infarction. Am J Med. 2013;126:789-97. [PubMed: 23856021]

9. Sarkisian L, Saaby L, Poulsen TS et al. Clinical Characteristics and Outcomes of Patients with Myocardial Infarction, Myocardial Injury, and Nonelevated Troponins. Am J Med. 2016;129:446e5-446.e21. 
10. O'Neill DE, Southern DA, Norris CM, O’Neill BJ, Graham MM. Type 2 MI in Southern Alberta: A Descriptive Analysis. Can J Cardiol. 2014;30:S165-S166.

11. Smilowitz NR, Beckman JA, Sherman SE, Berger JS. Hospital Readmission After Perioperative Acute Myocardial Infarction Associated With Noncardiac Surgery Circulation 2018 (in press).

12. Lofthus DM, Khalili H, Raja VN et al. Accuracy of acute myocardial infarction clinical diagnosis and its implications. Int J Cardiol. 2015;186:54-6. [PubMed: 25804472]

13. Pandey A, Golwala H, Hall HM et al. Association of US Centers for Medicare and Medicaid Services Hospital 30-Day Risk-Standardized Readmission Metric With Care Quality and Outcomes After Acute Myocardial Infarction: Findings From the National Cardiovascular Data Registry/Acute Coronary Treatment and Intervention Outcomes Network Registry-Get With the Guidelines. JAMA Cardiol. 2017;2:723-731. [PubMed: 28445559] 
McCarthy et al.

Page 8

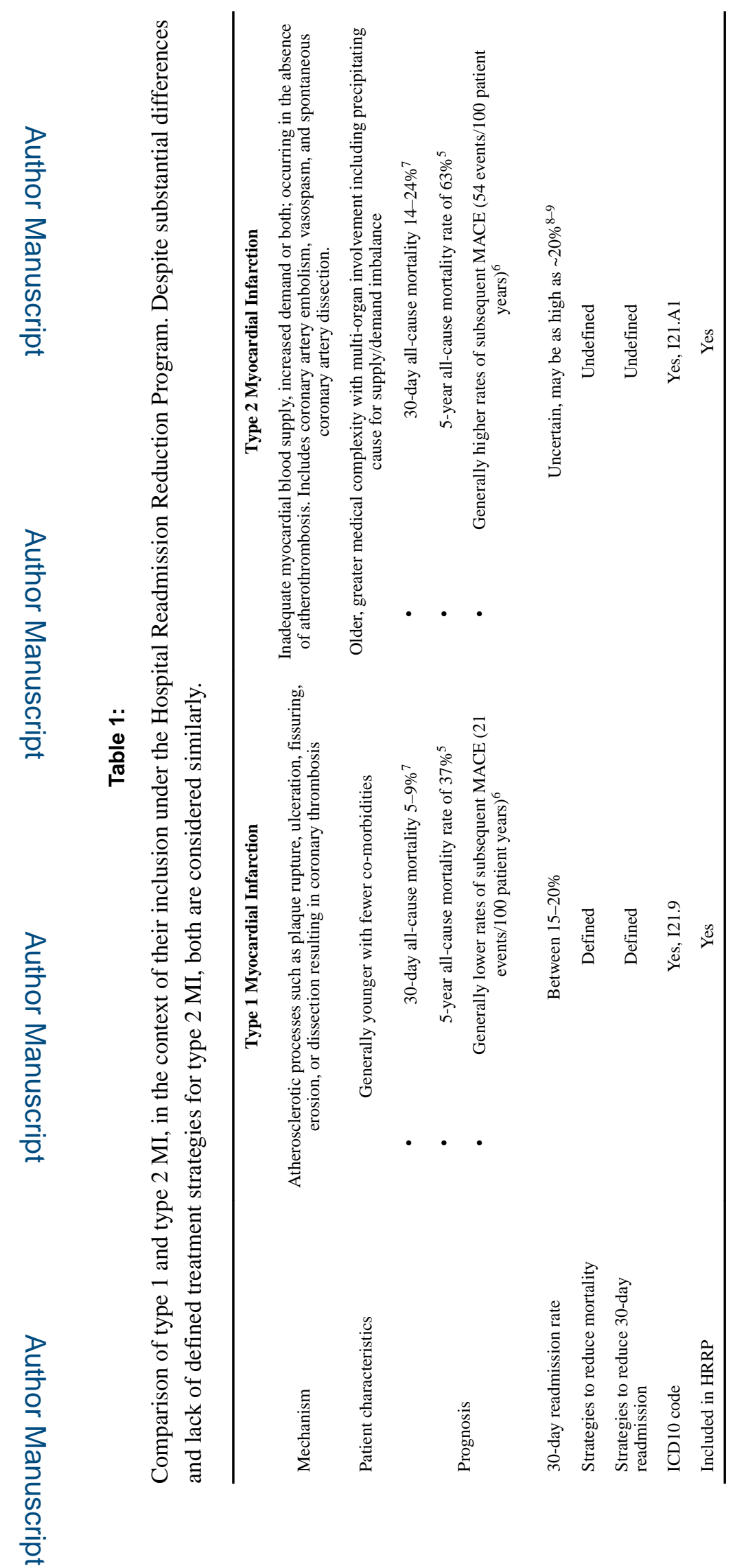

J Am Coll Cardiol. Author manuscript; available in PMC 2019 September 04. 